\title{
Liraglutide inhibits autophagy and apoptosis induced by high glucose through GLP-1R in renal tubular epithelial cells
}

\author{
X.ZHAO, G. LIU, H.SHEN, B.GAO, X. LI, J.FU, J.ZHOU and Q. JI \\ Department of Endocrinology, The First Affiliated Hospital of \\ The Fourth Military Medical University, Xi'an, Shaanxi, P.R. China
}

Received August 28, 2014; Accepted December 16, 2014

DOI: $10.3892 /$ ijmm.2014.2052

\begin{abstract}
Tubular atrophy and dysfunction is a critical process underlying diabetic nephropathy (DN). Understanding the mechanisms underlying renal tubular epithelial cell survival is important for the prevention of kidney failure associated with glucotoxicity. Autophagy is a cellular pathway involved in protein and organelle degradation. It is associated with many types of cellular homeostasis and human diseases. To date, little is known of the association between high concentrations of glucose and autophagy in renal tubular cells. In the present study, we investigated high glucose-induced toxicity in renal tubular epithelial cells by means of several complementary assays, including cell viability, cell death assays and changes in ultrastructure in an immortalized human kidney cell line, HK-2 cells. The extent of apoptosis was significantly increased in the HK-2 cells following treatment with high levels of glucose. In addition, in in vivo experiments using diabetic rats, high glucose exerted harmful effects on the tissue structure of the kidneys in the diabetic rats. Chronic exposure of the HK-2 cells and tubular epithelial cells of nephritic rats to high levels of glucose induced autophagy. Liraglutide inhibited these effects; however, treatment witht a glucagon-like peptide-1 receptor (GLP-1R) antagonist enhanced these effects. Our results also indicated that the exposure of the renal tubular epithelial cells to high glucose concentrations in vitro led to the downregulation of GLP-1R expression. Liraglutide reversed this effect, while the GLP-1R antagonist promoted it, promoting autophagy, suggesting that liraglutide exerts a renoprotective effect in the presence of high glucose, at least in part, by inhibiting autophagy and increasing GLP-1R expression in the HK-2 cells and kidneys of diabetic rats.
\end{abstract}

Correspondence to: Professor Jie Zhou or Professor Qiuhe Ji, Department of Endocrinology, The First Affiliated Hospital of The Fourth Military Medical University, No. 17 Changle West Road, Xi'an, Shaanxi 710032, P.R. China

E-mail: zhoujie@fmmu.edu.cn

E-mail: jiqiuhe@fmmu.edu.cn

Key words: high glucose, autophagy, autophagy-related gene, glucagon-like peptide-1 receptor, liraglutide

\section{Introduction}

The number of patients with diabetes is markedly increasing worldwide. Diabetes leads to vascular changes and dysfunction, as well as complications that result in increased morbidity and mortality (1). Among diabetic vascular complications, nephropathy contributes to the development of cardiovascular disease and is the leading cause of end-stage renal disease in developed countries $(2,3)$. Therefore, the prevention of renal insufficiency may improve the prognosis of patients with diabetes.

Numerous factors contribute to the development of diabetic nephropathy. Hyperglycemia alters both extracellular and intracellular metabolism, leading to effects such as glomerular hyperfiltration (4), oxidative stress (5), the accumulation of advanced glycation end products (AGEs) (6), the activation of protein kinase $\mathrm{C}$ (7), abnormal polyol metabolism (8), the overexpression of transforming growth factor- $\beta$ (TGF- $\beta$ ) (9) and inflammation. These effects have been recognized as classical characteristics of the pathogenesis of diabetic nephropathy. Additionally, intracellular stress associated with renal hypoxia $(10,11)$, reactive oxygen species (ROS) production by mitochondria (12-15) and endoplasmic reticulum (ER) stress (16-18) have recently been proposed as key mechanisms underlying the pathogenesis of diabetic nephropathy. Thus, the maintenance of cellular homeostasis against stress conditions may be a new therapeutic target for the treatment of diabetic nephropathy.

Autophagy is a tightly regulated process in which endogenous cellular proteins aggregate and damaged organelles are degraded by the lysosomal pathway. This process functions to maintain intracellular homeostasis and cell integrity. It has recently been highlighted as it can be stimulated by multiple types of cellular stressors, including starvation, hypoxia and ER stress. Emerging evidence also indicates that autophagy plays a critical role in several organs, particularly in highly metabolic organs, and that its alteration is involved in the pathogenesis of metabolic disease, immunity and autoimmunity, inflammation, development, aging and cancer $(19,20)$. It has been suggested that autophagy is enhanced and plays a protective role during kidney disease (21). Its renoprotective role in several animal models, including those used for aging and acute kidney injury, has also been demonstrated (22-24).

One possible mechanism through which autophagy protects cells is that it may eliminate damaged mitochondria. Autophagy 
involves the sequestration of proteins and cellular organelles into autophagosomes, which directs them to lysosomes (25). The formation of autophagosomes is dependent on the induction of several genes, including microtubule-associated protein 1 light chain 3 (LC3), Beclin1 and autophagy-related genes (Atgs) (26). Nonetheless, autophagy may also represent a form of programmed cell death known as autophagic cell death or type II programmed cell death, and altered autophagy is associated with the loss of renal tubular epithelial cell mass in diabetes. The role of autophagy in diabetic nephropathy remains a largely undetermined; thus, its underlying mechanisms are presently unclear.

Glucagon-like peptide-1 (GLP-1) is a gut incretin hormone and is currently considered an attractive agent for the treatment of type 2 diabetes. It has been shown to exert various beneficial effects on pancreatic $\beta$-cells, such as the enhancement of glucose-dependent insulin secretion (27), the acceleration of $\beta$-cell proliferation and the inhibition of $\beta$-cell apoptosis (28). In the gut and hypothalamus, GLP-1 has been shown to inhibit motility, gastric emptying (29) and the central regulation of feeding (30), resulting in the loss of body weight (27). It has previously been demonstrated that GLP-1 receptor (GLP-1R) is produced not only in the pancreas, gut and hypothalamus, but also in the kidneys (31). Liraglutide is a human incretinGLP-1 analogue with a high degree of homology to the native hormone. It shares $97 \%$ sequence identity with native human GLP-1. A recent study demonstrated that liraglutide inhibited cytokine-induced apoptosis in primary rat islet cells in a dose-dependent manner and reduced free fatty acid-induced apoptosis by approximately $50 \%$ (32).

In the present study, we evaluated the effects of high glucose concentrations on the induction of autophagy in the human renal tubular epithelial cell line, HK-2 cells, as well as in the kidneys of diabetic rats. We also investigated the ability of GLP-1 to protect HK-2 cells and diabetic rat kidneys against apoptosis induced by high glucose levels by targeting autophagy. As shown by our results, autophagy plays a potential role in nephropathy, which affects cell viability through the GLP-1 receptor.

\section{Materials and methods}

Cell culture. The human renal tubular epithelial cell line (HK-2) was purchased from the American Type Culture Collection (ATCC, Manassas, VA, USA) and was cultured in Dulbecco's modified Eagle's medium (DMEM) supplemented with $5.5 \mathrm{mM}$ D-glucose [normal glucose (N)], 10\% fetal bovine serum (FBS), epidermal growth factor, $100 \mathrm{U} / \mathrm{ml}$ penicillin, and $100 \mu \mathrm{g} / \mathrm{ml}$ streptomycin (all from HyClone, Logan, UT, USA) in a $5 \% \mathrm{CO}_{2}$ incubator at $37^{\circ} \mathrm{C}$.

3-(4,5-Dimethylthiazol-2-yl)-5-(3-carboxymethoxyphenyl)-2(4-sulfophenyl)-2H-tetrazolium (MTS) assay. The HK-2 cells were plated on 96-well plates at a density of $1.2 \times 10^{5}$ cells $/ \mathrm{cm}^{2}$ in medium containing either $5.5,16.7,25$ or $40 \mathrm{mM}$ glucose [high glucose (HG)] and incubated for up to $72 \mathrm{~h}$. Liraglutide (LG) was administrated at various concentrations (1, 10 and $100 \mathrm{nM}$ ) or HG with LG with glucagon-like peptide-1 receptor (GLP-1R) antagonist \{exendin-(9-39) [EX-(9-39)]; 1,000 nM\}. At 24,48 and $72 \mathrm{~h}$, cell viability was assessed by the ability of metabolically active cells to reduce the tetrazolium salt to formazan compounds using MTS reagent (Promega, Madison, WI, USA). The absorbance of the samples was measured using a microplate reader at a $450-\mathrm{nm}$ wavelength after $3 \mathrm{~h}$ of incubation with MTS solution $(0.19 \mathrm{mg} / \mathrm{ml})$. The results are expressed as the means \pm standard error of the mean (SEM) and are representative of 3 independent experiments.

Trypan blue exclusion assay. The cells were plated on 12-well plates at a density of $1.2 \times 10^{5}$ cells $/ \mathrm{cm}^{2}$ in medium containing either 5.5 or $40 \mathrm{mM}$ glucose, collected and stained with trypan blue (Invitrogen, Carlsbad, CA, USA). The total number of total cells, as well as the number of trypan blue-stained cells were counted using a hemocytometer (Cat. no. 02-671-6; Hausser Scientific, Horsham, PA, USA).

Western blot analysis. The cells were harvested and lysed in RIPA buffer (150 mM sodium chloride, $1.0 \%$ Triton X-100, $0.5 \%$ sodium deoxycholate, $0.1 \%$ sodium dodecyl sulfate, $50 \mathrm{mM}$ Tris, $\mathrm{pH}$ 8.0) with protease and phosphatase inhibitors, and centrifuged at $12,000 \mathrm{rpm}$ for $20 \mathrm{~min}$ at $4^{\circ} \mathrm{C}$. Protein samples were then mixed with loading buffer and boiled at $95-100^{\circ} \mathrm{C}$ for $5 \mathrm{~min}$. Total protein extracts were subjected to $12 \%$ SDS-PAGE. The separated proteins were transferred ono polyvinylidene fluoride membranes (Bio-Rad, Hercules, CA, USA) by electrotransfer. The blots were subsequently blocked with 5\% (v/v) skimmed milk (Nacalai Tesque, Kyoto, Japan) and incubated with GLP-1R rabbit antibody (ab39072, 1:1,000; Abcam, Cambridge, MA, USA), LC3 rabbit antibody (12741S, 1:1,000) and Beclin1 rabbit antibody (3495S, 1:1,000) (both from Cell Signaling Technology, Danvers, MA, USA) for $12 \mathrm{~h}$ at $4^{\circ} \mathrm{C}$, or with glyceraldehyde 3-phosphate dehydrogenase (GAPDH) antibody (G8795, 1:1,000; Sigma-Aldrich; St. Louis, MO, USA) for $1 \mathrm{~h}$ at room temperature. The membranes were incubated with horseradish peroxidase-linked, goat anti-rabbit (sc-2054) or anti-mouse IgG (sc-2055, 1:5,000; both from Santa Cruz Biotechnology, Inc., Santa Cruz, CA, USA) at room temperature for $2 \mathrm{~h}$. The blots were then visualized using a western blotting detection system (ECL plus; GE Healthcare, Princeton, NJ, USA).

Reverse transcription-quantitative (real-time) polymerase chain reaction ( $R T-q P C R)$. Total RNA was extracted from each sample using the Total RNA kit I (Omega Bio-Tek, Norcross, GA, USA). cDNA was synthesized from the individual samples of $1 \mu \mathrm{g}$ of total RNA using the PrimeScript ${ }^{\circledR}$ RT reagent kit (Takara Bio, Inc., Tokyo, Japan). Following the addition of each set of primers (final concentration, $0.4 \mu \mathrm{mol} / \mathrm{l}$ ) and template DNA to the master mix, quantitative (real-time) PCR was performed using a LightCycler (Roche Diagnostics, Tokyo, Japan) and SYBR Premix Ex Taq (Takara Bio, Inc.). The PCR protocol was as follows: initial denaturation $\left(95^{\circ} \mathrm{C}\right.$ for $30 \mathrm{sec})$ followed by 40 cycles of denaturation $\left(95^{\circ} \mathrm{C}\right.$ for $30 \mathrm{sec}, 60^{\circ} \mathrm{C}$ for $30 \mathrm{sec}$ and $72^{\circ} \mathrm{C}$ for $30 \mathrm{sec}$ ) and annealing and extension $\left(72^{\circ} \mathrm{C}\right.$ for $\left.1 \mathrm{~min}\right)$. The specific oligonucleotide primers were designed by Takara Bio Inc., and the primer sequences were as follows: GLP-1R sense, TCAAGGTCAACGG CTTATTAGTGAA and antisense, CCCAAGTGATGCAAG CAGAG; GAPDH sense, GCAC CGTCAAGGCTGAGAAC and antisense, TGGTGAAGACGCCAGTGGA. To visualize gene expression, individual DNA fragments were electro- 
phoresed on a $2 \%(\mathrm{w} / \mathrm{v})$ agarose gel (Sigma-Aldrich) and treated with ethidium bromide. cDNA of the human pancreas (Takara Bio, Inc.) was used as a positive control.

Electron microscopy. The HK-2 cells were plated in $0.01 \%$ poly-L-lysine (Sigma-Aldrich)-coated glass slides at a density of $1.5 \times 10^{4}$ cells/slide (area, $1.8 \mathrm{~cm}^{2}$ ), cultured for 1 day, and finally treated for $72 \mathrm{~h}$ in medium containing either a 5.5 or $40 \mathrm{mM}$ glucose concentration. The cells were fixed in $2.5 \%$ glutaraldehyde (Electron Microscopy Sciences, Hatfield, PA, USA) for $2 \mathrm{~h}$ and dissolved in $0.1 \mathrm{M}$ phosphate buffer (PB; $\mathrm{pH}$ 7.4). The HK-2 cells were then post-fixed for $1 \mathrm{~h}$ in $1 \%$ osmium tetroxide in PB and then stained with $70 \%$ ethanol containing 1\% uranyl acetate (Sigma-Aldrich). The HK-2 cells were then dehydrated in a graded alcohol series and embedded in epon (Sigma-Aldrich). Ultrathin sections (with silver to gray interference) were cut using a diamond knife (Diatome, Biel, Switzerland), mounted on Formvar-coated single-slot grids and then counterstained with $3 \%$ uranyl acetate and then with $0.2 \%$ lead citrate (Sigma-Aldrich). The sections were visualized under a Philips CM100 transmission electron microscope (Philips Electron Optics, Hillsboro, OR, USA).

Animals. The animal care, handling and in vivo studies complied with the guidelines provided by the Animal Care Committee of the Fourth Military Medical University. Male Sprague-Dawley rats (4 weeks old) were divided into the following groups: i) nondiabetic group $(\mathrm{n}=10)$; ii) diabetic group $(\mathrm{n}=10)$; iii) diabetic group treated with liraglutide $(\mathrm{n}=10)$; and iv) diabetic group treated with liraglutide and the GLP-1R antagonist, exendin-(9-39) (synthesized by Sigma-Aldrich; $n=10$ ). At the age of 5 weeks, the mice in the diabetic groups were administered intravenous injections of streptozotocin (MP Biomedicals, Santa Ana, CA, USA) at $60 \mathrm{mg} / \mathrm{kg}$ body weight in citrate buffer (pH 4.5). Only the rats with blood glucose concentrations $>16.7 \mathrm{mM}$ at 3 and 7 days following the injection of streptozotocin were included in the diabetic groups. The non-diabetic groups were administered injections of citrate buffer alone.

The groups treated with liraglutide were administered liraglutide (Novo Nordisk, Copenhagen, Denmark) subcutaneously at the dose of $0.3 \mathrm{mg} / \mathrm{kg} / 12 \mathrm{~h}$ for 5 weeks as previously described (33). Twice-daily dosing was used as the pharmacokinetic half-life of liraglutide is only approximately $4 \mathrm{~h}$ in rats. The mice in the group treated with liraglutide and the GLP-1R antagonist, exendin-(9-39), were subcutaneously administered liraglutide at the dose of $0.3 \mathrm{mg} / \mathrm{kg} / 12 \mathrm{~h}$ and exendin-(9-39) at the dose of $25 \mathrm{nmol} / \mathrm{kg} / 12 \mathrm{~h}$ for 5 weeks, beginning at 1 week after the streptozotocin or citrate buffer injections. The placebo groups were administered water alone using the same schedule as for the liraglutide treatment groups. All rats were allowed free access to standard food and tap water. All rats were euthanized at 5 weeks after the induction of diabetes in the diabetic groups, and the kidneys were weighed and fixed in $10 \%(\mathrm{v} / \mathrm{v})$ formalin or frozen in liquid nitrogen.

Assays of metabolic variables. Serum creatinine and blood urea nitrogen levels were measured using a BioMajesty JCA-BM12 analyzer (Hitachi, Tokyo, Japan). Body weight was monitored weekly from 4 weeks of age. Food intake was calculated as an average over a period of 3 days.
Immunohistochemistry. Hematoxylin and eosin (H\&E) and immunoperoxidase staining were performed as previously described (34). The kidneys were fixed in 10\% formaldehyde, and embedded in paraffin. Paraffin sections were cut at $3 \mu \mathrm{m}$ and deparaffinized for staining. Briefly, for immunoperoxidase staining, the primary antibodies used were GLP-1R rabbit antibody (1:200; Abcam) and LC3 rabbit antibody (1:200; Cell Signaling Technology), and were applied for $12 \mathrm{~h}$ at $4^{\circ} \mathrm{C}$. Secondary antibodies were biotin-labeled anti-rabbit IgG (Santa Cruz Biotechnology, Inc.), which were applied for $60 \mathrm{~min}$ at room temperature. The sections were counterstained with hematoxylin before being examined under a light microscope (Olympus, Tokyo, Japan).

Statistical analysis. Data are presented as means \pm SEM. Statistical analysis was assessed by one-way ANOVA followed by the least significant difference (LSD) t-test or Tamhane's T2 for multiple comparisons. A P-value $<0.05$ was considered to indicate a statistically significant difference.

\section{Results}

Chronic exposure to high glucose concentrations decreases cell proliferation and induces apoptosis. DMEM containing $5.5 \mathrm{mM}$ glucose has been used as a standard culture medium for HK-2 human renal tubular epithelial cells. In order to examine the cytotoxicity resulting from exposure to high glucose concentrations, cell viability was first measured by MTS assay, which is dependent on the metabolic activity of viable cells. The HK-2 cells were cultured in medium containing 5.5, 16.7, 25 or $40 \mathrm{mM}$ glucose for $72 \mathrm{~h}$. Cell viability significantly decreased in a glucose concentration-dependent manner (Fig. 1A). Of note, the HK-2 cells cultured in $40 \mathrm{mM}$ glucose for $24 \mathrm{~h}$ at a density of $1.2 \times 10^{5}$ cells $/ \mathrm{cm}^{2}$ showed increased viability. After $72 \mathrm{~h}$ of culture in $40 \mathrm{mM}$ glucose, however, there was a significant decrease in viability as compared to the cells incubated in medium with $5.5 \mathrm{mM}$ glucose (Fig. 1B).

The decrease in MTS values observed with $40 \mathrm{mM}$ glucose may be due to reduced proliferation or increased cell death. When cell death was assessed by the trypan blue exclusion assay, increased numbers of dead cells were observed at $72 \mathrm{~h}$ in the cells cultured with $40 \mathrm{mM}$ glucose (Fig. 1C).

Effects of liraglutide on $\mathrm{HK}-2$ cell viability are mediated through GLP-1R. The HK-2 cells cultured in a high concentration of glucose for $72 \mathrm{~h}$ showed a significantly decreased cell viability. Liraglutide significantly enhanced cell viability in a dose-dependent manner. Additionally, the effects of liraglutide were significantly blocked by exendin-(9-39), a GLP-1R antagonist (Fig. 1D). The loss of viability that occurred in the cells at $72 \mathrm{~h}$ cultured in the presence of 5.5 or $40 \mathrm{mM}$ glucose was associated with the activation of the common executioner caspase, caspase-3 at the protein level. Liraglutide downregulated caspase-3 expression, and exendin-(9-39) partly blocked this effect (Fig. 1E).

Effect of liraglutide on GLP-1R expression in HK-2 cells. The HK-2 cells stimulated with a high concentration of glucose for $72 \mathrm{~h}$ showed significantly decreased levels of GLP-1R expression. Liraglutide significantly enhanced GLP-1R 
A

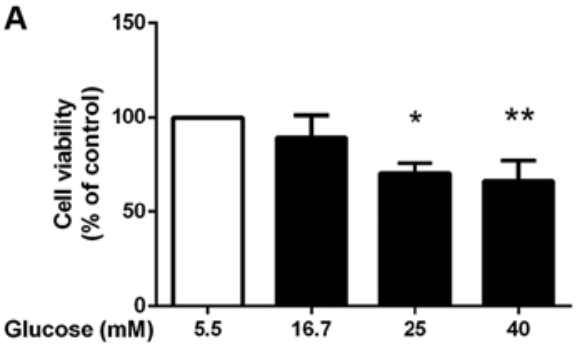

C

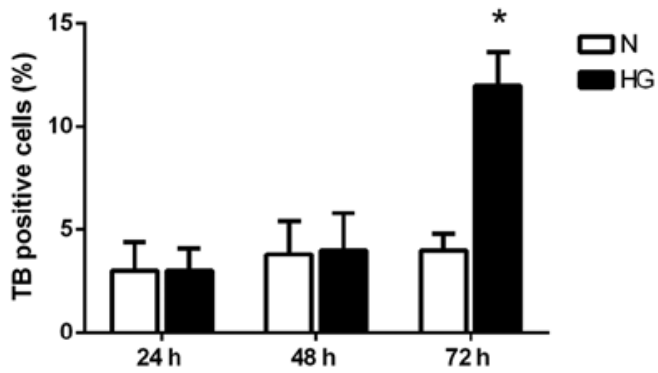

E

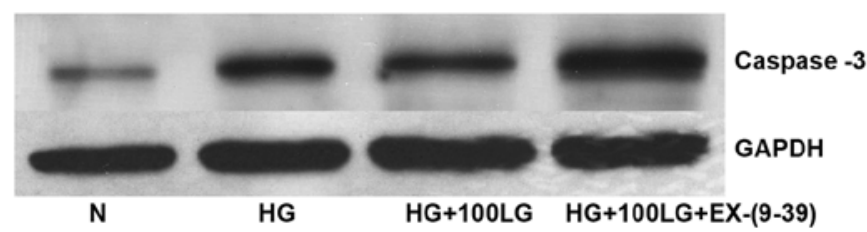

B $\left.{ }^{150}\right] \quad$ * $\quad$ 每

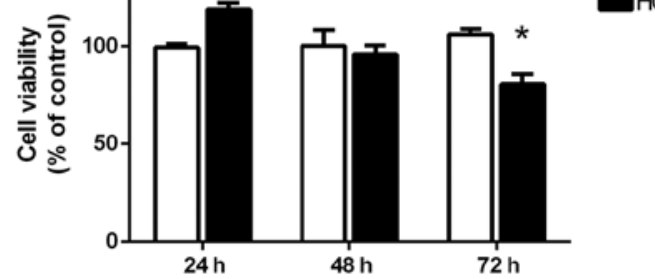

D

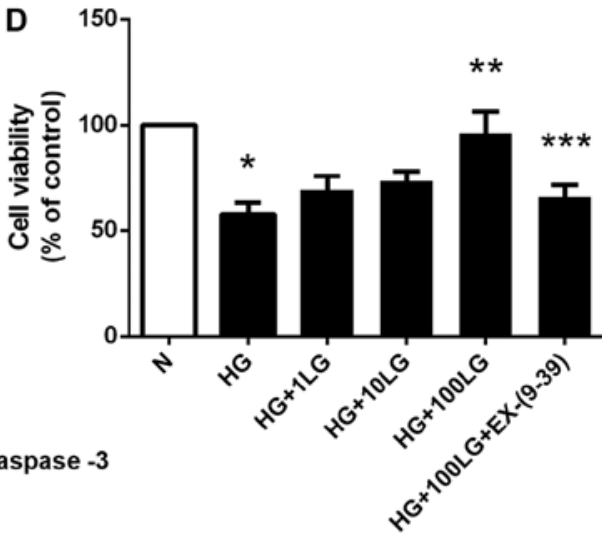

Figure 1. Chronic exposure to high levels of glucose (HG) decreases cell proliferation and induces apoptosis. (A) Cell viability was assessed by MTS assay in HK-2 cells culture in DMEM containing 5.5, 16.7, 25 or $40 \mathrm{mM}$ glucose for $72 \mathrm{~h}$. ${ }^{*} \mathrm{P}<0.05$ and ${ }^{* *} \mathrm{P}<0.01$ vs. $5.5 \mathrm{mM}$ group. (B) Cell viability was assessed by MTS assay in HK-2 cells cultured in DMEM containing 5.5 [normal glucose (N) group] or $40 \mathrm{mM}$ glucose [high glucose (HG) group] for 24 , 48 or $72 \mathrm{~h}$. ${ }^{*} \mathrm{P}<0.05$ vs. the corresponding sample from the $\mathrm{N}$ group at each time point. (C) Trypan blue (TB) exclusion assay was performed as a measurement of cell death. ${ }^{*} \mathrm{P}<0.05$ vs. normal glucose group at each time point. (D) Cell viability was assessed by MTS assay in HK-2 cells. Cells were cultured in medium containing 5.5 or $40 \mathrm{mM}$ glucose (HG) or HG with liraglutide (LG) at various concentrations (1, 10 and $100 \mathrm{nM})$ or HG with LG with glucagon-like peptide-1 receptor (GLP- 1R) antagonist $\{$ exendin-(9-39) [EX-(9-39)]; $1,000 \mathrm{nM}\}$ for $72 \mathrm{~h} .{ }^{*} \mathrm{P}<0.001 \mathrm{vs}$. N; ${ }^{* *} \mathrm{P}<0.001 \mathrm{vs}$. HG; ${ }^{* * *} \mathrm{P}<0.05 \mathrm{vs}$. HG $+100 \mathrm{LG}$. Values are the means \pm standard error of the mean (SEM). (E) Protein levels of the cleaved, active form of caspase-3 and glyceraldehyde 3-phosphate dehydrogenase (GAPDH) as an internal control were determined in HK-2 cells cultured in 5.5 or $40 \mathrm{mM}$ glucose medium or HG with LG or HG with LG with GLP-1R antagonist EX-(9-39).

mRNA expression in a dose-dependent manner. Additionally, the effects of liraglutide were significantly blocked by exendin-(9-39). The protein expression of GLP-1R was altered in a similar manner (Fig. 2).

Chronic exposure to high glucose concentrations alters cellular ultrastructure. Changes in the ultrastructure of HK-2 cells induced by high glucose were examined under a transmission electron microscope. No changes in cellular nuclei or membranes were observed in the HK-2 cells cultured in the presence of high glucose at $24 \mathrm{~h}$ (Fig. 3A). A large number of free-standing membrane structures and double-membrane vacuoles was observed in the cytoplasm after $48 \mathrm{~h}$, which resembled pre-autophagosomal structures. Typical apoptotic changes, with chromatin condensation and nuclear fragmentation, were observed in the cells exposed to high glucose concentrations, and the accumulation of glycogen significantly increased after $48 \mathrm{~h}$. Nuclear fragmentation and disappearance and the formation of a large number of vacuoles indicated cytoplasmic vacuolization (Fig. 3B).

High glucose concentrations promote autophagy in $\mathrm{HK}-2$ cells. The promotion of autophagy by exposure of the HK-2 cells to high glucose was confirmed by electron microscopy, showing an accumulation of autophagosomes (Fig. 3C and D, black arrows).

The HK-2 cells stimulated with a high concentration of glucose for $72 \mathrm{~h}$ exhibited an increased expression of the autophagic markers, LC3-II and Beclin1. Liraglutide significantly attenuated the increase in LC3-II and Beclin1 gene expression in a dose-dependent manner. Additionally, the effects of liraglutide were significantly blocked by the GLP-1R antagonist, exendin-(9-39) (Fig. 4).

Metabolic variables. Body and organ weights are presented in Table I. The body weights of the rats in the diabetic groups at 5 weeks after the initiation of liraglutide treatment were significantly lower than those of the rats in the non-diabetic group. The weight of the kidneys in the diabetic rats, normalized to the body weight of the rats in the non-diabetic groups, was significantly higher than that of the rats in the non-diabetic group. No significant differences were observed among the different diabetic groups.

Food intake was significantly increased in the diabetic groups, but was decreased in the diabetic groups at 5 weeks after the initiation of liraglutide treatment compared with the 
Table I. Metabolic variables of the 4 groups of rats at 5 weeks after the induction of diabetes.

\begin{tabular}{lcccc}
\hline Characteristics & $\mathrm{N}$ & $\mathrm{DM}$ & $\mathrm{DM}+\mathrm{LG}$ & $\mathrm{HG}+\mathrm{LG}+\mathrm{EX}-(9-39)$ \\
\hline Baseline & & & & \\
$\quad$ Body weight $(\mathrm{g})$ & $127 \pm 4$ & $131 \pm 5$ & $128 \pm 3$ & $129 \pm 3$ \\
Fasting blood glucose (mmol/l) & $4.0 \pm 0.3$ & $3.9 \pm 0.2$ & $3.7 \pm 0.3$ & $3.3 \pm 0.3$ \\
Four weeks after treatment & & & & $205 \pm 10^{\mathrm{a}}$ \\
Body weight (g) & $345 \pm 11$ & $221 \pm 13^{\mathrm{a}}$ & $28.5 \pm 4.6^{\mathrm{c}}$ & $209 \pm 18^{\mathrm{a}}$ \\
Food intake (g/day) & $25.9 \pm 4.3$ & $41.6 \pm 4.3^{\mathrm{b}}$ & $20.5 \pm 3.7^{\mathrm{b}}$ & $31.3 \pm 1.8$ \\
Fasting blood glucose (mmol/l) & $4.8 \pm 0.4$ & $26.7 \pm 4.2^{\mathrm{a}}$ & $10.6 \pm 0.4^{\mathrm{a}}$ & $20.3 \pm 4.3^{\mathrm{b}}$ \\
Relative kidney weight (g/kg) & $6.1 \pm 0.2$ & $12.1 \pm 0.4^{\mathrm{a}}$ & $18.0 \pm 1.4^{\mathrm{a}}$ & $10.8 \pm 0.5^{\mathrm{a}}$ \\
Blood urea nitrogen (mg/l) & $9.0 \pm 1.2$ & $20.9 \pm 2.3^{\mathrm{b}}$ & $63.9 \pm 3.1$ & $16.6 \pm 2.2$ \\
Creatinine (mmol/l) & $62.1 \pm 1.8$ & $74.4 \pm 4.5^{\mathrm{b}}$ & $67.3 \pm 2.9$ \\
\hline
\end{tabular}

Values are the means $\pm \mathrm{SEM} ; \mathrm{n}=10$ animals in the each group. ${ }^{\mathrm{a}} \mathrm{P}<0.001$ and ${ }^{\mathrm{b}} \mathrm{P}<0.05$ vs. non-diabetic group; ${ }^{\mathrm{c}} \mathrm{P}<0.05$ vs. diabetic group. SEM, standard error of the mean. N, normal glucose; DM, diabetic group; DM + LG, diabetic group treated with liraglutide; HG + LG + EX-(9-39), group treated with high glucose and liraglutide and exendin-39.

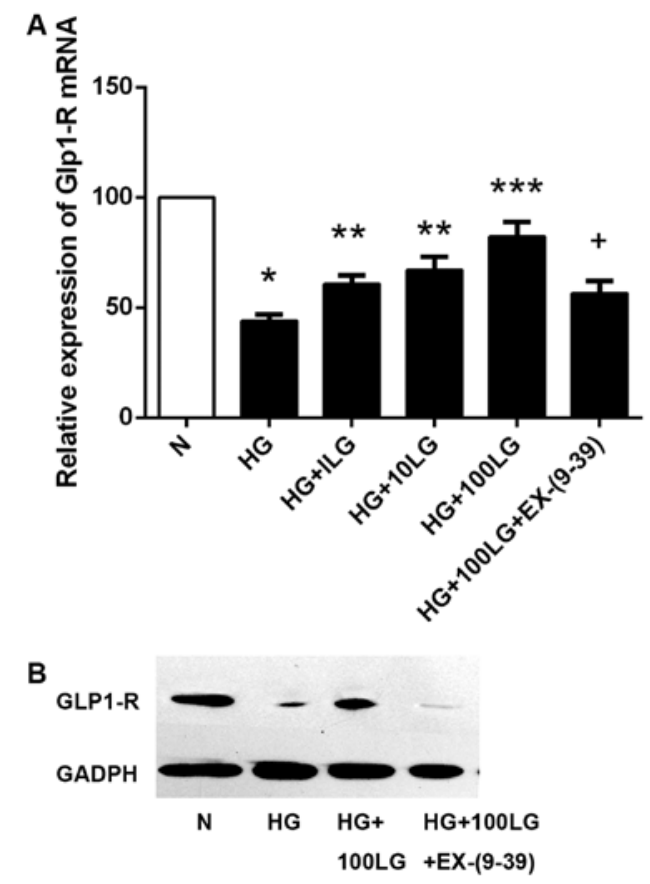

Figure 2.Effect of liraglutide(LG) on glucagon-like peptide-1 receptor(GLP-1R) expression in HK-2 cells. (A) Quantification of GLP-1R mRNA expression in HK-2 cells cultured in 5.5 [normal glucose $(\mathrm{N})$ ] or $40 \mathrm{mM}$ glucose medium or high glucose (HG) with LG at various concentrations $(1,10$ and $100 \mathrm{nM})$ or HG with LG with GLP-1R antagonist \{exendin-(9-39) [EX-(9-39)]\} by RT-qPCR. Values [means \pm standard error of the mean (SEM)] are presented as the fold change relative to glyceraldehyde 3-phosphate dehydrogenase (GAPDH). The experiment was repeated 3 times. ${ }^{*} \mathrm{P}<0.001$ vs. $\mathrm{N} ;{ }^{* * *} \mathrm{P}<0.001$ vs. $\mathrm{HG} ;{ }^{* * * *} \mathrm{P}<0.05$ vs. HG; ${ }^{+} \mathrm{P}<0.05$ vs. HG +100 LG. (B) Protein levels of GLP-1R in HK-2 cells cultured in 5.5 or $40 \mathrm{mM}$ glucose medium or HG with LG or HG with LG with GLP-1R antagonist EX-(9-39) by western blot analysis.

diabetic groups not receiving liraglutide. Serum creatinine and blood urea nitrogen levels, which are markers of renal injury, progressively increased in the diabetic groups during the experiment. Liraglutide treatment significantly reduced serum creatinine levels, but not the blood urea nitrogen levels, compared to the values in the diabetic groups not treated with liraglutide at 5 weeks.

Kidney morphology. The level of glomerular hypertrophy was significantly greater in the diabetic groups (Fig. 5B) than in the non-diabetic group (Fig. 5A). By contrast, liraglutide treatment inhibited glomerular hypertrophy in the rats in the diabetic group (Fig. 5C). The renal interstitium showed a significantly greater level of tubular hypertrophy and vacuolar degeneration in the diabetic groups than in the non-diabetic group. Liraglutide treatment improved this situation; however, its effects were blocked by exendin-(9-39) (Fig. 5D).

High glucose concentrations promote autophagy in the kidneys of diabetic rats. Immunohistochemistry revealed that the levels of the autophagic marker, LC3-II, were significantly upregulated in the kidneys of the diabetic rats (Fig. 6B) compared to those of the rats in non-diabetic group (Fig. 6A). Similar to the results from the in vitro experiments, liraglutide significantly decreased the expression of LC3-II (Fig. 6C). Additionally, the effects of liraglutide were partially blocked by exendin-(9-39) (Fig. 6D).

\section{Discussion}

The balance between the survival and death of renal tubular epithelial cells plays a crucial role in the pathogenesis of diabetic nephropathy. In the present study, we characterized high glucose-induced toxicity in renal tubular epithelial cells both in vivo and in vitro by means of several complementary assays, including cell viability, cell death assays and the assessment of the changes in the ultrastructure of HK-2 cells. The viability of renal tubular epithelial cells was markedly reduced and the number of dead cells, including those undergoing apoptosis, was significantly increased when the cells were treated for a prolonged period of time with high glucose concentrations. 


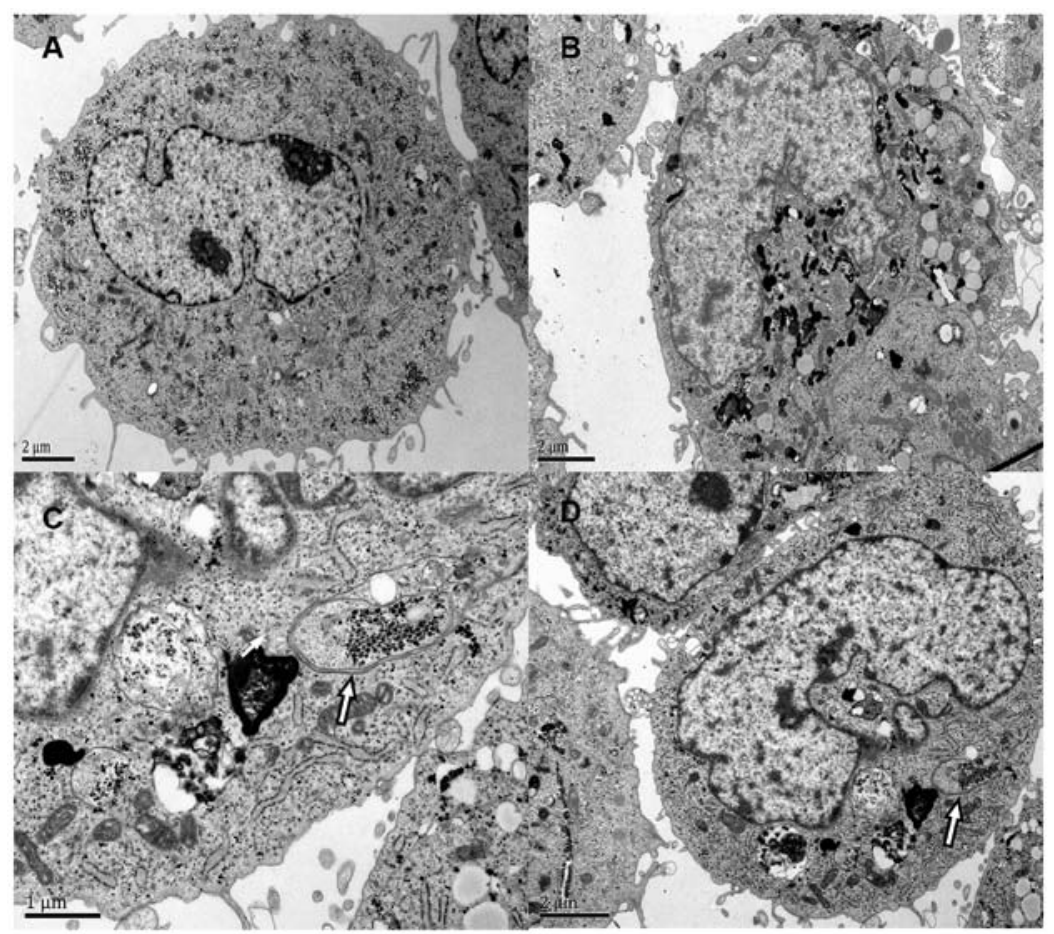

Figure 3. Changes in the ultrastructure of HK-2 cells induced by high glucose. (A) Controls. No changes in cellular nuclei or membranes were observed. (B) Changes associated with apoptosis following exposure to high glucose for $48 \mathrm{~h}$. Chromatin condensation and nuclear fragmentation were observed. (C and D) Autophagosomes of cells treated with high glucose for $72 \mathrm{~h}$. A large number of free-standing membrane structures and double-membrane vacuoles was observed (arrows).
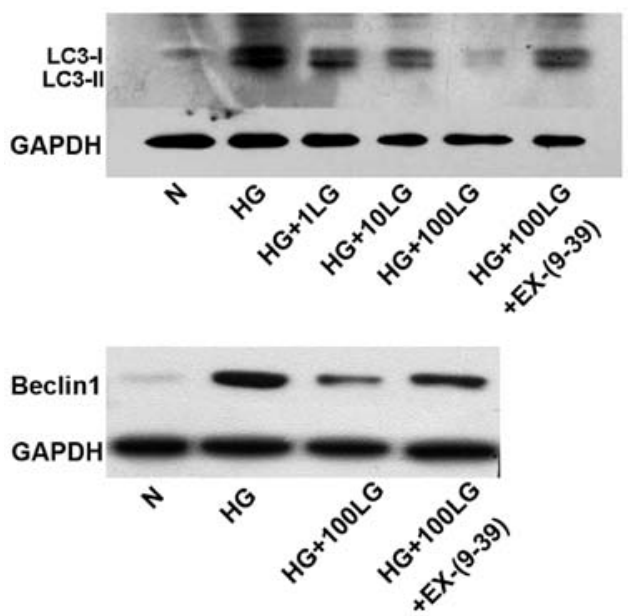

Figure 4. Changes in the expression of autophagic markers in HK-2 cells induced by high glucose (HG). Protein levels of microtubule-associated protein 1 light chain 3 (LC3; LC3-I and LC3-II) and Beclin1 in HK-2 cells stimulated with $40 \mathrm{mM} \mathrm{HG}$ or HG with liraglutide (LG) or HG with LG with glucagon-like peptide-1 receptor (GLP-1R) antagonist [EX-(9-39); 1,000 nM]

The exposure of renal tubular epithelial cells to high glucose concentrations for $72 \mathrm{~h}$ in vitro resulted in the downregulation of GLP-1R expression. Treatment with liraglutide increased cell viability, while exendin-(9-39) reversed this effect. These results support the concept that the changes in cell viabiltiy are caused by the reversible effects of glucotoxicity. These findings are consistent with the hypothesis that the unresponsiveness to GLP-1 is partly due to the changes in its receptor, suggesting that liraglutide has a renoprotective function that at least partially involves an increase in GLP-1R expression.
Importantly, we demonstrated that the chronic exposure of renal tubular epithelial cells and the kidneys of nephritic rats to elevated glucose levels induced autophagy. Autophagy is a cellular pathway involved in protein and organelle degradation. It occurs at a basal level in the majority of cells and is important in cellular homeostasis and responses to human disease (35). Autophagy can be induced by a variety of conditions, including nutrient deprivation and growth-factor depletion and hypoxia. However, little is known of the mechanisms underlying glucose-induced autophagy in renal tubular epithelial cells. Electron microscopy, the gold standard for monitoring the formation of autophagosomes, was used to demonstrate an elevated autophagic activity in HK-2 cells, where abundant vacuolization, a widely known morphologic indicator for autophagic cell death, was observed after 72-h of incubation in mediujm containing a high glucose concentration.

The pro-autophagic effect of high glucose was associated with the expression of LC3-II. The formation of autophagosomes includes the formation of pre-autophagosomal membranes (phagophore or isolation membrane), which is initiated by a class III phosphoinositide 3-kinase (PI3K) complex that includes Beclin1, and the elongation of the isolation membrane, which is stimulated by two ubiquitin-like conjugation systems (Atg12-Atg5 and LC3-phosphatidyletha nolamine) $(36,37)$. It has been demonstrated that the activation of Beclin1 is consistently associated with the induction of autophagy in cancer cells (38). LC3, which is associated with the control of autophagosome elongation, is the second essential ubiquitin-like protein. Cytosolic LC3-I is recruited to the membrane and interacts with phosphatidylethanolamine and is converted to LC3-II. Thus, LC3-II has been used as a marker of autophagy (39). In the present study, autophagy was induced 

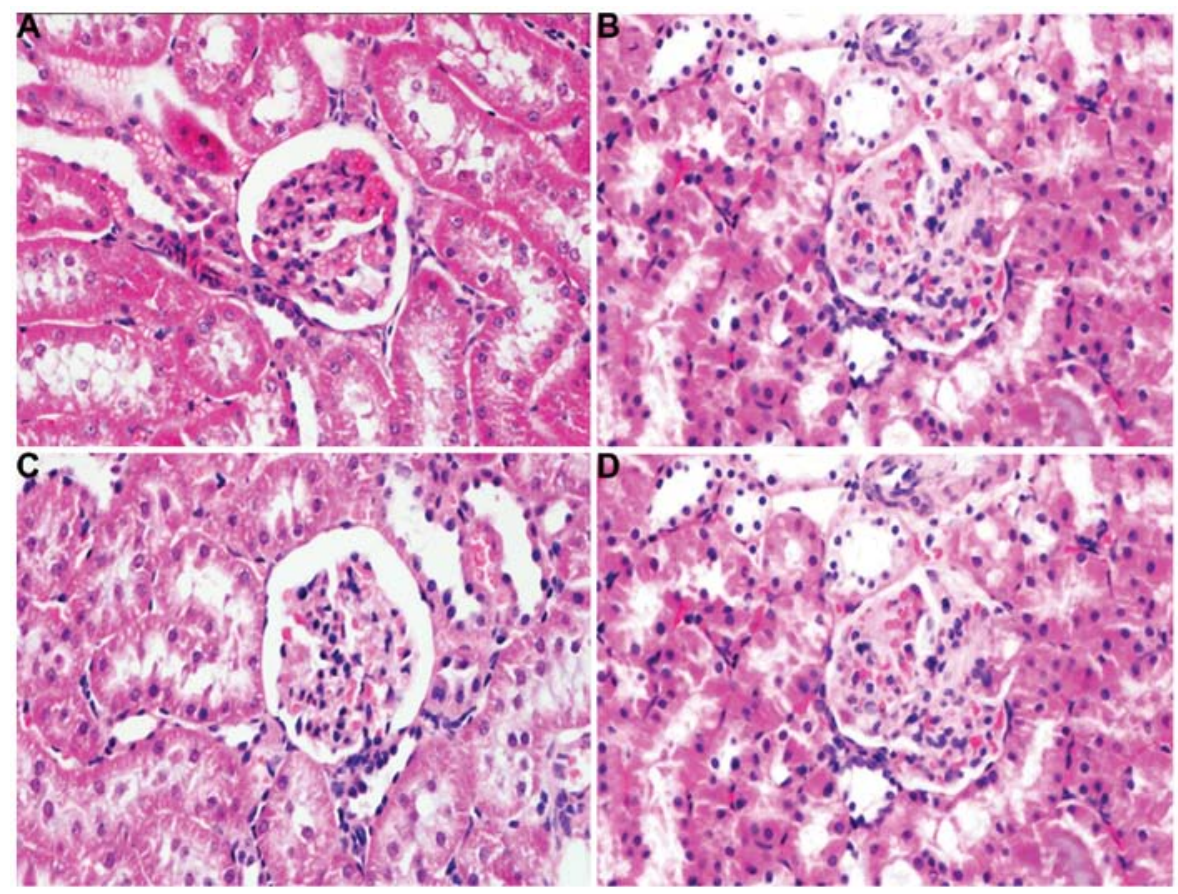

Figure 5. Effect of liraglutide on kidney morphology. Kidney morphology: (A) Non-diabetic group. Normal glomerular. (B) Diabetic group; glomerular hypertrophy was observed significantly. (C) Diabetes + liraglutide group. Liraglutide treatment inhibited glomerular hypertrophy in the rats. (D) Diabetes + liraglutide + exendin-(9-39) group. The effects of liraglutide were blocked by \{exendin-(9-39) [EX-(9-39)]\}. Glomerular hypertrophy was observed. Magnification for all images, $\mathrm{x} 400$.

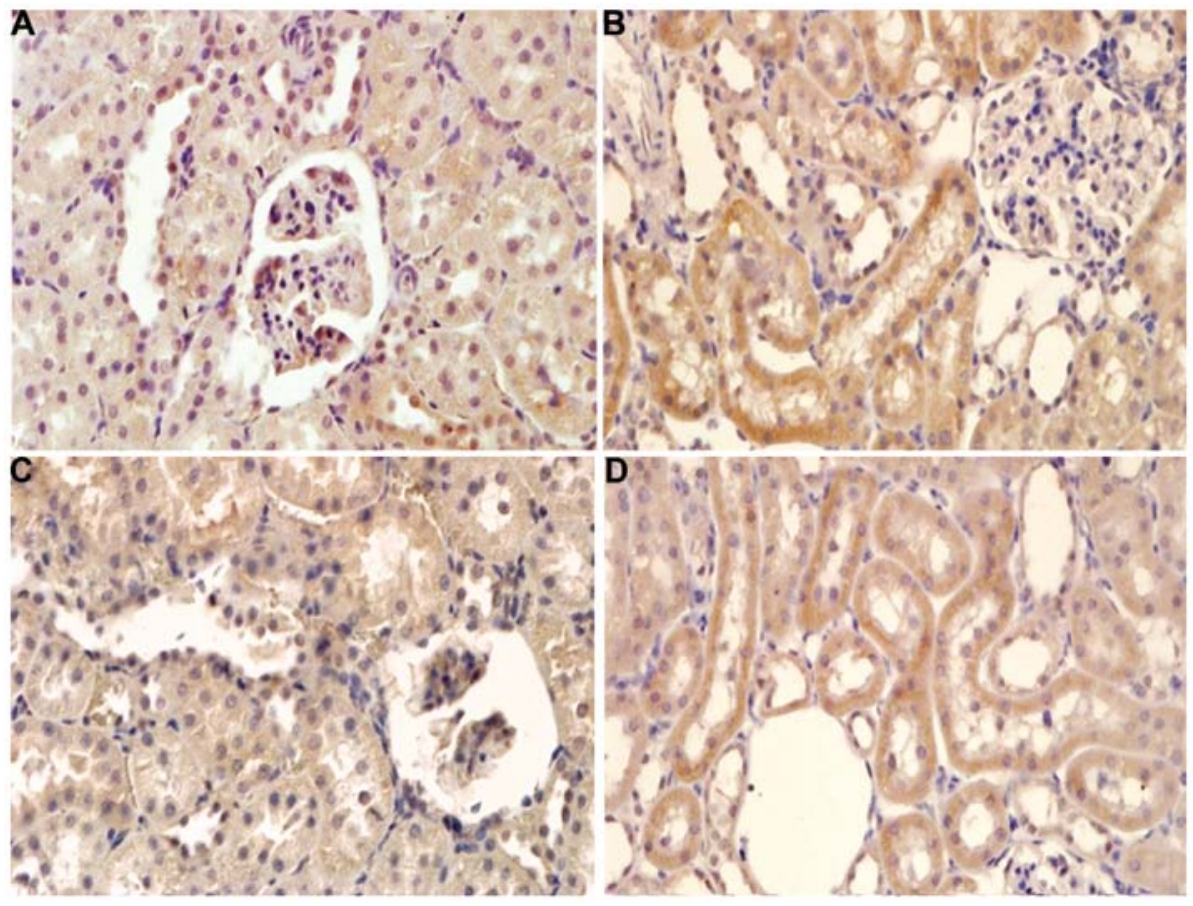

Figure 6. Effect of liraglutide on LC3 expression in rat kidneys. Immunoperoxidase staining for LC3: (A) Non-diabetic group. LC3 expression was at a basal level. (B) Diabetic group. The LC3 expression increased significantly. (C) Diabetic + liraglutide group. The liraglutide could decrease the LC3 expression; (D) Diabetic + liraglutide + exendin-(9-39) group. The effects of liraglutide were blocked by \{exendin-(9-39) [EX-(9-39)]\}. The LC3 expression increased slightly. Magnification for all images, $\mathrm{x} 400$.

by high glucose concentrations, as directly observed by the accumulation of autophagic vacuoles in the cytoplasm, as well as by the increase in the content of the autophagic markers, LC3-II and Beclin1. The prominent role of autophagy in the response of renal tubular epithelial cells to prolonged exposure to high glucose concentrations suggests that autophagy may be an effective therapeutic target for the treatment of diabetic nephropathy.

The addition of liraglutide to the high-glucose medium significantly increased the viability of the HK-2 cells that were exposed to high glucose alone. Liraglutide is a human incretin and GLP-1 analogue with high homology to the native 
hormone. The intestinal absorption of glucose stimulates its secretion, which increases insulin and decreases glucagon secretion (27). GLP-1 is associated with enhanced satiety, reduced food intake and weight loss. GLP-1 preserves $\beta$-cell morphology and function, and reduces cellular apoptosis (40). In the present study, we also found that liraglutide inhibited the apoptosis of HK-2 cells, which was accompanied by a significant decrease in autophagy, and that exendin-(9-39), a GLP-1R antagonist, blocked the cytoprotective effects of liraglutide. The same trends in cell viability, GLP-1R expression and autophagy suggested the potential connections in this pathological procedure of high glucose-induced toxicity in renal tubular epithelial cells.

The question of whether autophagy is the driver of cell death or a pro-survival process in response to certain stress conditions remains controversial. Although autophagy was initially described as a cytoprotective mechanism under conditions of nutrient deprivation, several lines of evidence have indicated a role for autophagy in promoting cell death (41-43), similar to the in vivo and in vitro results obseved in the present study. This indicates that liraglutide may also prevent injury in renal tubular epithelial cells by reducing the autophagy induced by exposure to high glucose and may be a promising therapeutic agent. Future studies are required to determine the precise mechanisms underlying the differential effects of liraglutatide on apoptosis and autophagy, and to optimize its cytoprotective effects.

\section{Acknowledgements}

This study was supported by the grants from the National Science Foundation of China, Grant No. 30870948 and 81102006. The authors thank all the staff at the Department of Endocrinology, The First Affiliated Hospital of The Fourth Military Medical University, Xi'an, P.R. China for their assistance with this project. The authors declare no potential conflicts of interest with respect to the authorship and/or publication of this article.

\section{References}

1. Chen L, Magliano DJ and Zimmet PZ: The worldwide epidemiology of type 2 diabetes mellitus - present and future perspectives. Nat Rev Endocrinol 8: 228-236, 2011.

2. de Zeeuw D, Parving HH and Henning RH: Microalbuminuria as an early marker for cardiovascular disease. J Am Soc Nephrol 17: 2100-2105, 2006.

3. Go AS, Chertow GM, Fan D, McCulloch CE and Hsu CY: Chronic kidney disease and the risks of death, cardiovascular events, and hospitalization. N Engl J Med 351: 1296-1305, 2004.

4. Magee GM, Bilous RW, Cardwell CR, Hunter SJ, Kee F and Fogarty DG: Is hyperfiltration associated with the future risk of developing diabetic nephropathy? A meta-analysis. Diabetologia 52: 691-697, 2009.

5. Baynes JW: Role of oxidative stress in development of complications in diabetes. Diabetes 40: 405-412, 1991.

6. Brownlee M, Cerami A and Vlassara H: Advanced glycosylation end products in tissue and the biochemical basis of diabetic complications. N Engl J Med 318: 1315-1321, 1988.

7. Koya D, Jirousek MR, Lin YW, Ishii H, Kuboki K and King GL: Characterization of protein kinase $\mathrm{C}$ beta isoform activation on the gene expression of transforming growth factor-beta, extracellular matrix components, and prostanoids in the glomeruli of diabetic rats. J Clin Invest 100: 115-126, 1997.

8. Dunlop M: Aldose reductase and the role of the polyol pathway in diabetic nephropathy. Kidney Int Suppl 77: S3-S12, 2000.
9. Ziyadeh FN, Sharma K, Ericksen M and Wolf G: Stimulation of collagen gene expression and protein synthesis in murine mesangial cells by high glucose is mediated by autocrine activation of transforming growth factor-beta. J Clin Invest 93: 536-542, 1994

10. Singh DK, Winocour P and Farrington K: Mechanisms of disease: the hypoxic tubular hypothesis of diabetic nephropathy. Nat Clin Pract Nephrol 4: 216-226, 2008.

11. Miyata T and de Strihou Cv: Diabetic nephropathy: a disorder of oxygen metabolism? Nat Rev Nephrol 6: 83-95, 2010.

12. Giacco F and Brownlee M: Oxidative stress and diabetic complications. Circ Res 107: 1058-1070, 2010.

13. Kashihara N, Haruna Y, Kondeti VK and Kanwar YS: Oxidative stress in diabetic nephropathy. Curr Med Chem 17: 4256-4269, 2010.

14. Hakim FA and Pflueger A: Role of oxidative stress in diabetic kidney disease. Med Sci Monit 16: RA37-RA48, 2010.

15. Ha H, Hwang IA, Park JH and Lee HB: Role of reactive oxygen species in the pathogenesis of diabetic nephropathy. Diabetes Res Clin Pract 82 (Suppl 1): S42-S45, 2008.

16. Wu J, Zhang R, Torreggiani M, et al: Induction of diabetes in aged C57B6 mice results in severe nephropathy: an association with oxidative stress, endoplasmic reticulum stress, and inflammation. Am J Pathol 176: 2163-2176, 2010.

17. Qi W, Mu J, Luo ZF, et al: Attenuation of diabetic nephropathy in diabetes rats induced by streptozotocin by regulating the endoplasmic reticulum stress inflammatory response. Metabolism 60: 594-603, 2011.

18. Cybulsky AV, Takano T, Papillon J, Kitzler TM and Bijian K: Endoplasmic reticulum stress in glomerular epithelial cell injury. Am J Physiol Renal Physiol 301: F496-F508, 2011.

19. Masini M, Bugliani M, Lupi R, et al: Autophagy in human type 2 diabetes pancreatic beta cells. Diabetologia 52: 1083-1086, 2009.

20. Ebato C, Uchida T, Arakawa M, et al: Autophagy is important in islet homeostasis and compensatory increase of beta cell mass in response to high-fat diet. Cell Metab 8: 325-332, 2008.

21. Hartleben B, Gödel M, Meyer-Schwesinger C, et al: Autophagy influences glomerular disease susceptibility and maintains podocyte homeostasis in aging mice. J Clin Invest 120: 1084-1096, 2010.

22. Kimura T, Takabatake Y, Takahashi A, et al: Autophagy protects the proximal tubule from degeneration and acute ischemic injury. J Am Soc Nephrol 22: 902-913, 2011.

23. Jiang M, Liu K, Luo J and Dong Z: Autophagy is a renoprotective mechanism during in vitro hypoxia and in vivo ischemia-reperfusion injury. Am J Pathol 176: 1181-1192, 2010.

24. Kume S, Uzu T, Horiike K, et al: Calorie restriction enhances cell adaptation to hypoxia through Sirt1-dependent mitochondrial autophagy in mouse aged kidney. J Clin Invest 120: 1043-1055, 2010.

25. Ohsumi Y and Mizushima N: Two ubiquitin-like conjugation systems essential for autophagy. Semin Cell Dev Biol 15: 231-236, 2004.

26. Xie Z and Klionsky DJ: Autophagosome formation: core machinery and adaptations. Nat Cell Biol 9: 1102-1109, 2007.

27. Baggio LL and Drucker DJ: Biology of incretins: GLP-1 and GIP. Gastroenterology 132: 2131-2157, 2007.

28. Farilla L, Hui H, Bertolotto C, et al: Glucagon-like peptide-1 promotes islet cell growth and inhibits apoptosis in Zucker diabetic rats. Endocrinology 143: 4397-4408, 2002.

29. Nauck MA, Niedereichholz U, Ettler R, et al: Glucagon-like peptide 1 inhibition of gastric emptying outweighs its insulinotropic effects in healthy humans. Am J Physiol 273: E981-E988, 1997.

30. Turton MD, O'Shea D, Gunn I, et al: A role for glucagon-like peptide-1 in the central regulation of feeding. Nature 379: 69-72, 1996.

31. Bullock BP, Heller RS and Habener JF: Tissue distribution of messenger ribonucleic acid encoding the rat glucagon-like peptide-1 receptor. Endocrinology 137: 2968-2978, 1996.

32. Wajcberg E and Amarah A: Liraglutide in the management of type 2 diabetes. Drug Des Devel Ther 4: 279-290, 2010.

33. Hayes MR, Kanoski SE, Alhadeff AL and Grill HJ: Comparative effects of the long-acting GLP-1 receptor ligands, liraglutide and exendin-4, on food intake and body weight suppression in rats. Obesity (Silver Spring) 19: 1342-1349, 2011.

34. Sugimoto H, Shikata K, Hirata K, et al: Increased expression of intercellular adhesion molecule-1 (ICAM-1) in diabetic rat glomeruli: glomerular hyperfiltration is a potential mechanism of ICAM-1 upregulation. Diabetes 46: 2075-2081, 1997. 
35. Mizushima N, Levine B, Cuervo AM and Klionsky DJ: Autophagy fights disease through cellular self-digestion. Nature 451: 1069-1075, 2008.

36. Martinet W and De Meyer GR: Autophagy in atherosclerosis: a cell survival and death phenomenon with therapeutic potential. Circ Res 104: 304-317, 2009.

37. Zhang H, Kong X, Kang J, et al: Oxidative stress induces parallel autophagy and mitochondria dysfunction in human glioma U251 cells. Toxicol Sci 110: 376-388, 2009.

38. Kiyono K, Suzuki HI, Matsuyama H, et al: Autophagy is activated by TGF-beta and potentiates TGF-beta-mediated growth inhibition in human hepatocellular carcinoma cells. Cancer Res 69: 8844-8852, 2009.

39. Kawakami T, Inagi R, Takano H, et al: Endoplasmic reticulum stress induces autophagy in renal proximal tubular cells. Nephrol Dial Transplant 24: 2665-2672, 2009.
40. Marsh BJ, Soden C, Alarcón C, et al: Regulated autophagy controls hormone content in secretory-deficient pancreatic endocrine beta-cells. Mol Endocrinol 21: 2255-2269, 2007.

41. Law BY, Wang M, Ma DL, et al: Alisol B, a novel inhibitor of the sarcoplasmic/endoplasmic reticulum $\mathrm{Ca}(2+)$ ATPase pump, induces autophagy, endoplasmic reticulum stress, and apoptosis. Mol Cancer Ther 9: 718-730, 2010.

42. Zhu J, Wang KZ and Chu CT: After the banquet: mitochondrial biogenesis, mitophagy, and cell survival. Autophagy 9: 16631676, 2013.

43. Petersen M, Hofius D and Andersen SU: Signaling unmasked: Autophagy and catalase promote programmed cell death. Autophagy 10: 520-521, 2014. 